\title{
Apatinib with etoposide capsules as a third- or further-line therapy for extensive-stage small cell lung cancer: an open-label, multicenter, single-arm phase II trial
}

\author{
Zhen He ${ }^{1 \#}$, Hanqiong Zhou ${ }^{1 \#}$, Junsheng Wang ${ }^{2}$, Ding Li $^{3}$, Xudong Zhang ${ }^{4}$, Pengyuan Wang ${ }^{5}$, \\ Tianjiang $\mathrm{Ma}^{6}$, Yueqiang Zhang ${ }^{7}$, Chuntao Tian ${ }^{8}$, Yunfang Chen ${ }^{9}$, Minglei Zou ${ }^{10}$, Yu Han ${ }^{11}$, Cong $\mathrm{Xu}^{1}$, \\ Shuxiang $\mathrm{Ma}^{1}$, Lili Wang ${ }^{1}$, Xuan $\mathrm{Wu}^{1}$, Gongbin Chen ${ }^{12}$, Qiming Wang ${ }^{1}$ \\ ${ }^{1}$ Department of Internal Medicine, Henan Cancer Hospital, Affiliated Cancer Hospital of Zhengzhou University, Zhengzhou, China; ${ }^{2}$ Department \\ of Medical Oncology, Anyang Cancer Hospital, Anyang, China; ${ }^{3}$ Department of Pharmacy, Affiliated Cancer Hospital of Zhengzhou University, \\ Henan Cancer Hospital, Zhengzhou, China; ${ }^{4}$ Department of Medical Oncology, The First Affiliated Hospital of Zhengzhou University, Zhengzhou, \\ China; ${ }^{5}$ Department of Medical Oncology, Xuchang Central Hospital, Xuchang, China; ${ }^{\circ}$ Department of Medical Oncology, Luohe Central Hospital, \\ Luohe, China; ${ }^{7}$ Department of Medical Oncology, Zhoukou Chinese Medicine Hospital, Zhoukou, China; ${ }^{8}$ Department of Medical Oncology, \\ Sanmenxia Central Hospital, Sanmenxia, China; ${ }^{9}$ Department of Medical Oncology, Zhumadian Central Hospital, Zhumadian, China; ${ }^{10}$ Department \\ of Medical Oncology, The Second People's Hospital of Jiaozuo, Jiaozuo, China; ${ }^{11}$ Department of Medical Oncology, Jiyuan People's Hospital of \\ Henan Province, Jiyuan, China; ${ }^{12}$ Department of Medical Oncology, Shangqiu first People's Hospital, Shangqiu, China \\ Contributions: (I) Conception and design: Q Wang, Z He, G Chen; (II) Administrative support: Q Wang, Z He, G Chen; (III) Provision of study \\ materials or patients: Q Wang, Z He; (IV) Collection and assembly of data: All authors; (V) Data analysis and interpretation: Z He, H Zhou, C Xu; (VI) \\ Manuscript writing: All authors; (VII) Final approval of manuscript: All authors. \\ "These authors contributed equally to this work as joint first authors. \\ Correspondence to: Qiming Wang, MD, PhD. Department of Internal Medicine, Affiliated Cancer Hospital of Zhengzhou University, Henan Cancer \\ Hospital, No. 127 Dongming Road, Zhengzhou 450008, China. Email: qimingwang1006@126.com; Gongbin Chen. Department of Medical \\ Oncology, Shangqiu first People’s Hospital, No. 292 Kaixuan South Road, Shangqiu, China. Email: chengongbin@sina.com.
}

Background: Patients with extensive-stage small-cell lung cancer (ES-SCLC) have a particularly poor prognosis. And the treatment options for patients with relapsed or refractory ES-SCLC are limited. Thus, we conducted an open-label, multicenter, single-arm phase II clinical trial to assess the efficacy and safety of apatinib plus etoposide capsules as the third- or further-line treatment in ES-SCLC patients.

Methods: Patients with ES-SCLC who experienced disease progression following 2 to 3 previous therapies from 11 medical centers in China were enrolled to receive apatinib (250 mg/d, continuously) and etoposide capsules $(50 \mathrm{mg} / \mathrm{d}$, on day $1-21$, per 28 days). The treatment continued until disease progression, treatment intolerance, or death. The primary endpoint was progression-free survival (PFS), and the secondary endpoints were objective response rate (ORR), overall survival (OS), and safety.

Results: Fifty-six patients with relapsed or refractory ES-SCLC were enrolled from January 2018 to February 2020 and 53 of them were eventually included in the evaluation population. The median followup was 9.8 months. At the data cut-off time (March 5, 2020), 39 patients (74\%) had died and $44(83 \%)$ had progressed. The median PFS was 3.0 months (95\% CI, 2.1-3.9) and the median OS was 5.0 months (95\% CI, 3.6-6.4). No complete responses were seen. Eleven patients (21\%) showed a best response of partial response and $37(70 \%)$ patients achieved stable disease. The ORR was 20.8\% (11/53), and the disease control rate (DCR) was 90.6\% (48/53). The 6-month OS rate was 40.1\% (95\% CI, 26.2-54). After 12 months, the OS rate was $18.4 \%$ (95\% CI, 4.7-32.1). Possible treatment-related grade III/IV adverse events included leukopenia [8 (15.1\%)], neutropenia [7 (13.2\%)], anemia [4 (7.4\%)], and hand-foot syndrome [2 (3.8\%)]. During the study, no mortality occurred as a consequence of treatment.

Conclusions: Apatinib combined with etoposide capsules exhibits efficacy and has an acceptable safety profile. It could be used as a later-line treatment for ES-SCLC patients who have been heavily pretreated 
with standard therapies. Further exploration of apatinib combined with etoposide capsules in phase III trials is warranted.

Keywords: Small cell lung cancer (SCLC); apatinib; etoposide capsules; efficacy; safety

Submitted Nov 06, 2020. Accepted for publication Jan 06, 2021.

doi: $10.21037 /$ tlcr-20-1235

View this article at: http://dx.doi.org/10.21037/tlcr-20-1235

\section{Introduction}

Small cell lung cancer (SCLC) comprises approximately $15 \%$ of lung cancers. Patients with SCLC typically exhibit rapid tumor growth and early metastatic dissemination. Diagnoses of SCLC are often made at an extensive stage and the prognosis is extremely poor (1). The survival rate of patients with extensive-stage SCLC (ES-SCLC) at 5 years after diagnosis does not exceed $7 \%$, and the median overall survival (OS) is a mere 9-11 months (2).

Although most patients are sensitive to the standard firstline chemotherapy of etoposide plus platinum (EP), with response rates of $60 \%$ to $70 \%$, almost every patient goes on to develop progressive disease or relapse with resistance to further therapy $(3,4)$. Generally, the clinical response to follow-up treatment is limited and is mainly dependent on the response time after the initial treatment. Patients whose disease relapses more than 3 months after the end of firstline treatment have a higher response rate than those whose disease relapses within 3 months (25\% vs. 10\%) $(3,5)$. The OS of the standard second-line chemotherapy topotecan is only approximately 30 weeks and alternative regimens for third- or further-line therapy are limited (6). Therefore, a novel treatment strategy for patients with refractory or relapsed ES-SCLC is urgently needed.

Sustained angiogenesis is a hallmark and essential alteration in the growth process of malignant tumors and represents a uniquely promising therapeutic target (7). Angiogenesis inhibitors, which include antivascular endothelial growth factor (VEGF) antibodies and multi-receptor tyrosine kinase inhibitors (TKIs), have shown anti-tumor activity in various cancers $(2,8,9)$. However, a meta-analysis showed that the combination of bevacizumab and chemotherapy as a first-line treatment for ES-SCLC demonstrated no significant survival benefit, which is consistent with the results of two clinical trials (10-12). However, a recent phase III study showed that bevacizumab combined with EP exhibited manageable toxicities and a significant improvement in progression-free survival (PFS) (13).

Apatinib, as a small molecule inhibitor of vascular endothelial growth factor receptor (VEGFR)-2 tyrosine kinase, can block the transmission of the VEGF/VEGFR-2 signaling pathway, and has shown efficacy against several cancers, including SCLC $(14,15)$. A retrospective study revealed that apatinib at a dose of $250 \mathrm{mg}$ displayed promise as an option for maintenance treatment in a Chinese ESSCLC cohort following the failure of etoposide plus platinum-containing chemotherapy (16). Despite etoposide plus platinum-based chemotherapy being used as the firstline treatment for patients with ES-SCLC for more than three decades (17), daily oral etoposide (50 $\mathrm{mg}$ once daily) was reported to be capable of producing a palliative effect and objective responses in heavily pretreated patients (18). Thus, apatinib (250 mg once daily) plus oral etoposide (50 mg once daily) could be a potential treatment strategy for heavily pretreated cancer patients. In the AEROC study, apatinib combined with etoposide capsules showed promising efficacy and safety in ovarian cancer patients with platinum-resistant or platinum-refractory disease (19). However, the efficacy and safety of apatinib plus etoposide capsules in patients with ES-SCLC as the third- or furtherline therapy is unclear. Therefore, we conducted this prospective, single-arm, phase II clinical trial to evaluate the efficacy and safety of apatinib combined with etoposide capsules as a third or further line of treatment for ESSCLC. We present the following article in accordance with the TREND reporting checklist (available at http://dx.doi. org/10.21037/tlcr-20-1235).

\section{Methods}

\section{Patients and study design}

We conducted the present phase II, open-label, multicenter, single-arm clinical trial at 11 Chinese medical centers and registered in a clinical trial registry (ClinicalTrials. gov identifier: NCT03389087). Patients between the 
ages of 18-75 years who had a diagnosis of ES-SCLC and had relapsed or progressed following second- or furtherline treatment, including the EP regimen, were eligible to participate in the study. Other inclusion criteria were: at least one measurable lesion according to the Response Evaluation Criteria in Solid Tumors (RECIST; version 1.1); an Eastern Cooperative Group performance status (ECOG PS) score of 0-2; a minimum predicted life expectancy of 3 months; and adequate bone marrow function (a white blood cell count of $\geq 3.5 \times 10^{3}$ cells $/ \mu \mathrm{L}$, an absolute neutrophil count of $\geq 1.5 \times 10^{3}$ cells $/ \mu \mathrm{L}$, a platelet count of $\geq 100 \times 10^{3}$ cells $/ \mu \mathrm{L}$, and a hemoglobin concentration of $\geq 90 \mathrm{~g} / \mathrm{L}$ ), adequate liver function [a total bilirubin level $\leq 1.5$ times the upper limit of normal (ULN), an aspartate transaminase (AST) level $\leq 2.5$ times the ULN, an alanine transaminase (ALT) level $\leq 2.5$ times ULN. An AST and ALT level $\leq 5$ times the ULN if the patient had hepatic metastasis], and adequate renal function (serum creatinine $\leq 1.25$ times the ULN or endogenous creatinine clearance $\geq 45 \mathrm{~mL} / \mathrm{min}$ ).

The main exclusion criteria included: prior treatment with apatinib or any angiogenesis inhibitors; active or newly diagnosed untreated metastatic lesions of the central nervous system (CNS) (not including brain metastases that were stable or treated at least 21 days before the start of the study); uncontrolled hypertension; clinically significant hemoptysis within the 2 months prior to the study; abnormal coagulant function; and significant bleeding tendency or signs of hemorrhage. Patients with any one or more of the following conditions were also excluded: myocardial infarction; uncontrolled arrhythmia; class III or IV congestive heart failure as defined by the New York Heart Association; arterial or venous thrombosis within the 12 months prior to day 1 of this study; undergoing current anticoagulant therapy; and proteinuria (defined as urine protein $\geq++$ or 24 -hour urinary protein $\geq 1.0 \mathrm{~g}$ ).

The independent ethics committee of Henan Cancer Hospital of Zhengzhou University granted approval for this study, which was carried out in adherence to the Declaration of Helsinki (as revise in 2013) and Good Clinical Practice Guidelines. All patients provided written informed consent.

\section{Procedures}

All patients received oral apatinib (at a dose of $250 \mathrm{mg}$, administered once daily on each day of a 28 -day cycle) combined with oral etoposide (at a dose of $50 \mathrm{mg}$ daily on days $1-21$ of a 28 -day cycle) treatment. Treatment was administered until the disease progressed, or the patient withdrew, developed intolerable toxicity, or died. Given the anti-tumor efficacy of apatinib at the dose of $250 \mathrm{mg}$ and etoposide at the dose of $50 \mathrm{mg}$ once daily, dose reductions were not permitted in our study. However, if uncontrollable adverse events occurred, dose interruption was allowed. In the event of a grade III or IV toxicity, treatment was delayed until the patient had recovered to grade I or better before resumption at the same dose. Repeated dose interruptions were permitted if the intolerable adverse events recurred.

Measurable lesions were assessed and documented prior to treatment. Computed tomography (CT) or magnetic resonance imaging (MRI) was used to assess tumor response according to RECIST 1.1 following each treatment cycle of apatinib combined with etoposide until disease progression was confirmed. Enhanced CT scans of the chest and upper abdomen were necessary after every cycle, and enhanced CT scans of the lower abdomen and pelvis or enhanced brain MRI were performed if related lesions were documented or new-onset associated symptoms occurred. As well as routine physical, urine, and stool examinations, the patients also underwent hematology, serum chemistry, vital signs, ECOG PS, 12-lead electrocardiogram, and blood pressure assessments after every cycle. In addition, routine blood monitoring was conducted weekly during treatment and 1 week before the start of treatment. If electrocardiography showed abnormal signs, myocardial enzymes were measured. Adverse events were evaluated and documented continuously throughout treatment according to the National Cancer Institute Common Terminology Criteria for Adverse Events version 4.0 (NCI-CTCAE v.4.0). The survival status of the patients was followed up every 3 months during treatment.

\section{Outcomes}

The primary endpoint was PFS, which was defined as the interval from the randomization date until the date of first documented progression or any-cause mortality. The secondary endpoints included OS, objective response rate (ORR), and safety. OS was defined as the interval from the randomization date until the date of any-cause mortality. The ORR was defined as the proportion of patients who achieved a complete response (CR) and partial response (PR) according to the RECIST evaluation criteria, version 1.1. Treatment safety was evaluated based on the occurrence of adverse events, with the severity of these events graded 


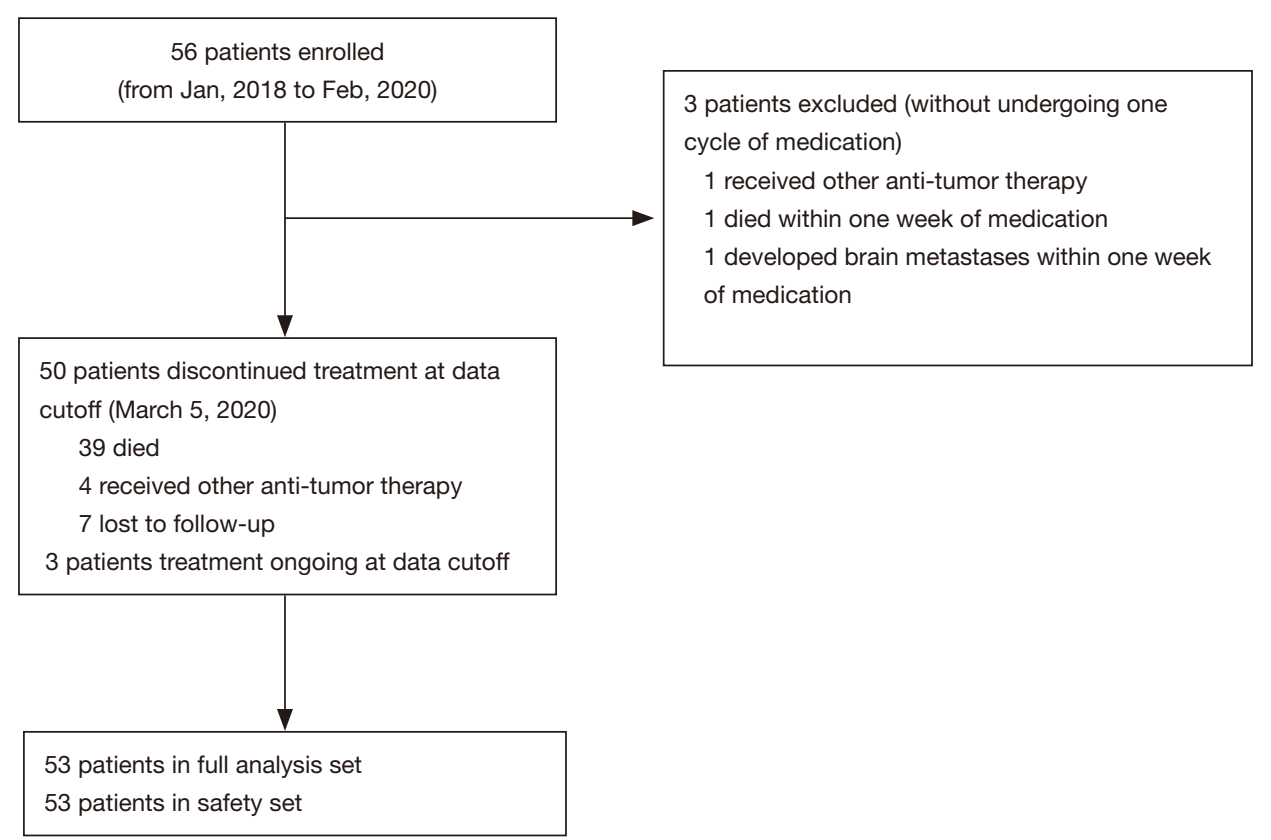

Figure 1 Trial profile. Three patients without undergoing one cycle of medication were excluded.

according to the NCI-CTCAE v.4.0.

\section{Statistical analyses}

PFS of 2 months was considered to be a clinically meaningful threshold for this study. Using Fisher's one-sided exact test in the PASS 11 software, we determined that a sample size of 37 patients would provide at least $80 \%$ power and, at most, a $5 \%$ probability of making a type I error. Taking a $20 \%$ drop-out rate into consideration, we planned to enroll 50 patients.

Kaplan-Meier curves were used to analyze PFS and OS. All statistical analyses were two-sided, and $\mathrm{P}<0.05$ indicated a statistically significant difference. All statistical analysis was performed with SPSS for Windows Version 23.0 (IBM, Chicago, IL, USA).

\section{Results}

\section{Patient characteristics}

Between January 2018 and February 2020, 56 patients with relapsed or refractory ES-SCLC from 11 Chinese medical centers were enrolled into our trial. Each patient was administered at least one dose of the combination treatment of oral apatinib plus etoposide. Three patients were excluded: one case died within the first cycle of treatment due to rapid disease progression; one case developed brain metastasis after 1 week of medication; and one case discontinued the treatment plan after 1 week of medication. Eventually, 53 patients who received the protocol treatment without any major protocol violations were included in the full analysis set, which comprised the per-protocol set and the safety analysis set (Figure 1).

Baseline characteristics of the 53 patients included in the full analysis set are listed in Table 1. These patients had a median age of 57 years (range, 30-77 years), and 40 (40/53; $75.5 \%)$ patients were male. In the full analysis set, 26 patients $(26 / 53,49.06 \%)$ were over the age of 60 years, and 45 patients $(45 / 53,84.9 \%)$ had a smoking history. Fourteen (14/53, 26.4\%), 10 (10/53, 18.9\%), and 10 (10/53, 18.9\%) patients presented with metastases of the bone, liver, and brain, respectively. Thirty-three patients $(33 / 53,62.3 \%)$ had received 2 prior lines of treatment, while 20 patients $(20 / 53,37.7 \%)$ had received $\geq 3$ prior lines of treatment.

\section{Efficacy evaluation}

As of March 5, 2020, 44 (83.0\%) of 53 patients had experienced disease progression events ( 9 patients had incomplete post-baseline efficacy assessments). Thirty-nine 
Table 1 Baseline patient characteristics

\begin{tabular}{|c|c|}
\hline Characteristics & All treated patients $(n=53)$ \\
\hline \multicolumn{2}{|l|}{ Sex, n (\%) } \\
\hline Male & $40(75.5)$ \\
\hline Female & $13(24.5)$ \\
\hline \multicolumn{2}{|l|}{ Age (years), n (\%) } \\
\hline Median [range] & $57[30-77]$ \\
\hline$<60$ & $27(50.9)$ \\
\hline$\geq 60$ & $26(49.1)$ \\
\hline \multicolumn{2}{|c|}{ ECOG performance status, n (\%) } \\
\hline $0-1$ & $49(92.5)$ \\
\hline 2 & $4(7.5)$ \\
\hline \multicolumn{2}{|c|}{ Smoking history, n (\%) } \\
\hline Yes & $45(84.9)$ \\
\hline No & $8(15.1)$ \\
\hline \multicolumn{2}{|c|}{ Previous lines of treatment, $\mathrm{n}(\%)$} \\
\hline 2 & $33(62.3)$ \\
\hline$\geq 3$ & $20(37.7)$ \\
\hline \multicolumn{2}{|c|}{ Brain metastases, n (\%) } \\
\hline Yes & $10(18.9)$ \\
\hline No & $43(81.1)$ \\
\hline \multicolumn{2}{|c|}{ Liver metastases, n (\%) } \\
\hline Yes & $10(18.9)$ \\
\hline No & $43(81.1)$ \\
\hline \multicolumn{2}{|c|}{ Bone metastases, n (\%) } \\
\hline Yes & $14(26.4)$ \\
\hline No & $39(73.6)$ \\
\hline
\end{tabular}

ECOG, Eastern Cooperative Oncology Group.

(73.6\%) of the 53 patients had died, 7 had survived, and 7 had been lost to follow-up; 3 of the surviving patients are still receiving the treatment. The best response was $\mathrm{PR}$ in 11 patients (21.0 \%) and SD in 37 patients (70\%) (Figure 2). The ORR was recorded for 11 (20.8\%) of 53 patients, with $\mathrm{PR}$ recorded in each case. The full analysis set had a median PFS reaching 3.0 months (95\% CI, 2.1-3.9), and 3 (5.7\%) of the 53 patients did not experience disease progression, with a PFS of 19.2, 9.2, and 4.9 months, respectively, as of the cutoff date. At the cutoff date, the median OS was 5.0 months (95\% CI, 3.6-6.4) (Figure 3). The OS rate was

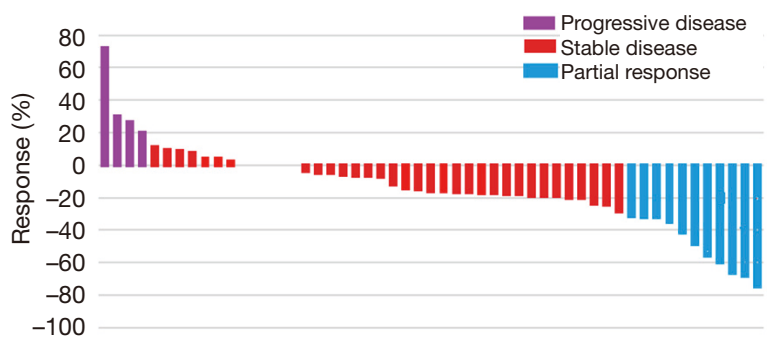

Figure 2 Waterfall plot showing the best response in target lesion size in 53 patients who had at least one post-baseline efficacy assessment. Five patients had $0 \%$ change from baseline. The color indicates the type of response.

$40.1 \%$ (95\% CI, 26.2-54) and 18.4\% (95\% CI, 4.7-32.1) at 6 and 12 months, respectively.

\section{Adverse events}

Toxicity is an important aspect of cancer treatment strategies. A previous study showed that apatinib at a dose of $750 \mathrm{mg}$ once a day resulted in substantial toxicities in a cohort of breast cancer patients. Most of the patients experienced toxicities of grade III or worse, which led to a dose delay with dose reduction (20). However, in previous studies, a low dose of apatinib (250 $\mathrm{mg}$ daily) as a monotherapy or combination therapy has shown tolerable or controllable toxicity in numerous cancers $(21,22)$. In this phase II study, we evaluated the safety of apatinib $(250 \mathrm{mg}$ once daily) plus etoposide capsules (50 mg once daily). The most common grade I or II adverse events observed in our study were anemia [17 (24.5\%)], leukopenia [16 (30.2\%)], fatigue [15 $(28.3 \%)]$, nausea [13 $(24.5 \%)]$, proteinuria [10 $(18.9 \%)]$, vomiting [10 (18.9\%)], hand-foot syndrome [9 $(17 \%)]$, thrombocytopenia [9 (17\%)], and hypertension [8 (15.1\%)] (Table 2). No treatment-related deaths or unexpected toxicities occurred.

Severe adverse events were observed in three patients. One patient had anemia with a hemoglobin concentration of $5.4 \mathrm{~g} / \mathrm{dL}$ and was transferred to hospital for a blood transfusion; this event was possibly related to the etoposide capsules. One patient had neutropenia with an absolute neutrophil count of 300 cells per $\mu \mathrm{L}$, which could possibly be attributed to the etoposide capsules. Another patient developed massive ascites as a result of disease progression.

Dose interruption was required by 11 patients due to intolerable toxicity including 8 patients with grade III or VI leukopenia, 1 patient with anemia, 1 patient with impaired 

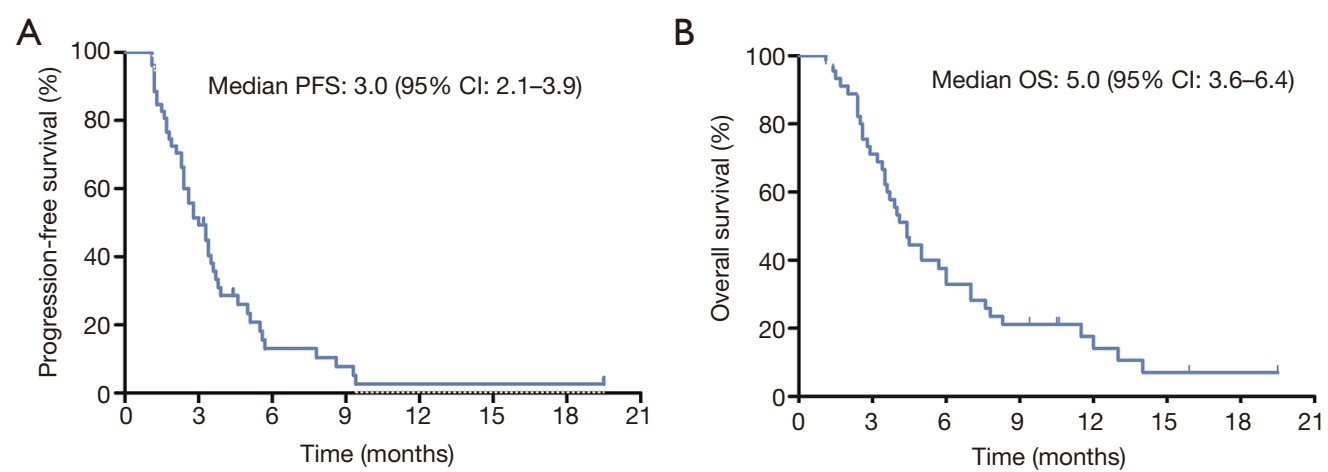

Figure 3 Kaplan-Meier curve for PFS in patients with at least one post-baseline efficacy assessment (n=53). Estimation of the PFS of patients with ES-SCLC treated with apatinib plus etoposide capsules (A). Estimation of the OS of patients with ES-SCLC treated with apatinib plus etoposide capsules (B).

liver function, and 1 patient with mucositis. Treatment was resumed at the same dosage after recovery to grade I or better.

\section{Discussion}

As a highly aggressive lung cancer, SCLC is defined by poor differentiation, rapid tumor growth, and poor survival outcome (23). Generally, SCLC is divided into limited and extensive stages depending on the extent of the disease. The International Association for the Study of Lung Cancer (IASLC) defines limited-stage patients as those without distant metastasis, and extensive-stage patients as those with distant metastatic sites (24). ES-SCLC makes up 60-70\% of all SCLCs and has a survival rate of only $1 \%$ at 5 years after diagnosis (25). Despite the extreme sensitivity SCLC shows to initial treatment with chemotherapy or radiotherapy, drug resistance eventually occurs, and most patients progress rapidly or relapse, which has remained an immense treatment challenge, despite the arrival of immunotherapy in recent years $(23,26,27)$.

In the past 30 years, etoposide combined with platinum has become broadly recognized as the first-line gold standard of chemotherapy for SCLC (23). Although SCLC is highly sensitive to chemotherapy, most patients develop recurrence or metastasis within 1 year after receiving the initial treatment. According to the duration time after first-line chemotherapy, the disease is classified into three categories: sensitive type (tumor response lasting 90 days or longer), resistant type (recurrence within 90 days of completing primary therapy), or refractory type (progression during the first-line chemotherapy) $(28,29)$. While some chemotherapeutics such as irinotecan, topotecan, and paclitaxel have exhibited anti-tumor activity in the secondline treatment of SCLC, their efficacy is mainly dependent on the patient's response to first-line chemotherapy and is still unsatisfactory, with toxic reactions including severe neutropenia and anemia (29). For patients who suffer relapse after second-line treatment, follow-up treatment strategies are limited.

Apatinib is a small-molecule TKI that can suppress the activity of VEGFR-2 to decrease cell proliferation and induce apoptosis. It has been shown to exert an antitumor effect in numerous solid tumors, including SCLC (14,30-32). Etoposide, an inhibitor of topoisomerase II, has demonstrated anti-cancer activity both in untreated and pretreated patients with ES-SCLC (33). Additionally, in a phase II, single-arm, prospective study, oral apatinib combined with etoposide capsules showed promising efficacy and reasonable toxicity in patients with ovarian tumors that were resistant or refractory to platinum (19). However, concern remains over the use of this combination regimen in ES-SCLC.

As far as we are aware, ours is the first evaluative study on the efficacy and safety of apatinib plus etoposide capsules in patients with relapsed or refractory ES-SCLC. This study reached all of its primary and secondary endpoints, and demonstrated considerable efficacy and reasonable safety of apatinib plus etoposide capsules in the 53 patients enrolled. An objective response was observed in almost $21 \%$ of cases. The median PFS reached 3.0 months (95\% CI, 2.1-3.9), and the median OS reached 5.0 months (95\% CI, 3.6-6.4). Moreover, this combination treatment had manageable toxicity. As apatinib and etoposide are orally administrated, 
Translational Lung Cancer Research, Vol 10, No 2 February 2021

Table 2 Possible treatment-related adverse events in the safety population

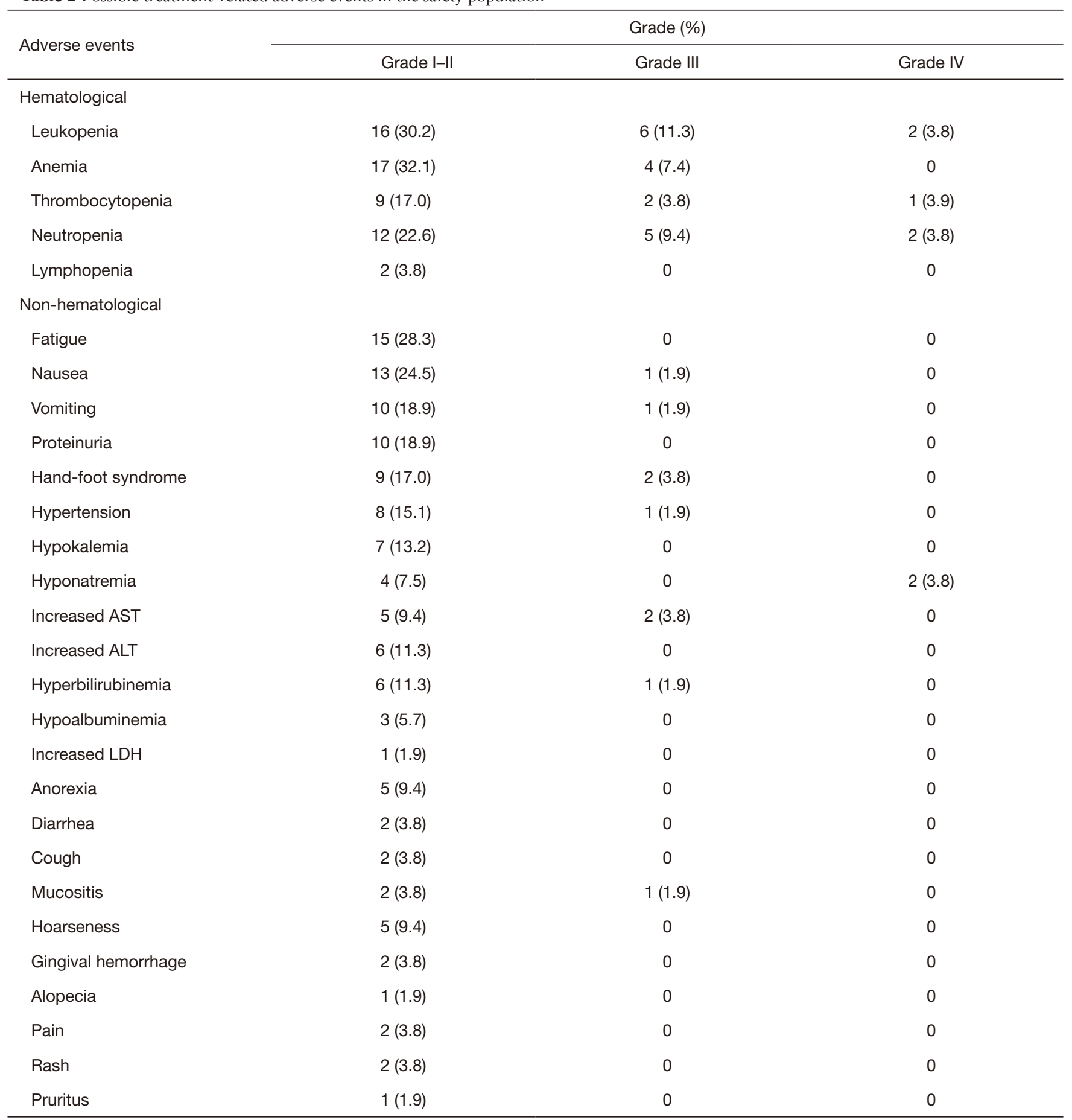

AST, aspartate aminotransferase; ALT, alanine aminotransferase; LDH, lactate dehydrogenase. 
intravenous transfusion or frequent hospital admission is not necessary; thus, this regimen may be conveniently administrated and economically beneficial for patients. Also, in most ES-SCLC patients, death occurs rapidly after disease progression in the third or further line setting, which means that oral medication might be more suitable for those receiving palliative therapy, especially during the COVID-19 pandemic, as it removes the need for frequent hospital visits (34).

According to the published reports, fatigue, hypertension, proteinuria, and hand-foot syndrome are the most frequently reported adverse events related to apatinib. These adverse events were also observed in our study, with patients experiencing fatigue (28.3\%), proteinuria (18.9\%), hand-foot syndrome (17\%), and hypertension (15.1\%), and most cases were grade I or II. Hoarseness was observed in 5 patients $(9.4 \%)$ in our study, which was considered to be related to the use of apatinib. Hand-foot syndrome (3.8\%), hypertension $(1.9 \%)$, and mucositis $(1.9 \%)$ were the main grade III or IV adverse events associated with apatinib, and leukopenia (15.1\%), neutropenia (13.2\%), and anemia (7.4\%) were the leading hematological toxicities resulting from etoposide. Grade IV hyponatremia was observed in two patients in our study and was possibly disease-related. All adverse events were tolerable after symptomatic and supportive treatment, and no serious adverse events, such as interstitial pneumonia, occurred. Previous studies have also reported that the toxicity of VEGFR-TKIs acts as a predictive biomarker of the therapeutic effect; however, in this study, no significant relationship was observed between adverse events and PFS or OS.

Nivolumab or pembrolizumab is approved by Food and Drug Administration (FDA) as a third- or later-line therapy for patients with ES-SCLC. In the CheckMate 032 study evaluating the efficacy and safety of third- or later-line nivolumab monotherapy treatment, an ORR of $11.9 \%$ was observed in $71.6 \%$ of ES-SCLC patients receiving thirdline treatment; the median PFS and OS were 1.4 months and 5.6 months, respectively; and the OS rate at 12 months was $28.3 \%$ (35). In the KEYNOTE-028 study, $50 \%$ of patients with ES-SCLC and a tumor cell, immune infiltrate and stromal summative programmed death ligand-1 status combined positive score (CPS) of $\geq 1 \%$ received pembrolizumab as the third line of treatment. The ORR for the entire cohort was $33 \%$. The median PFS and OS were 1.9 and 9.7 months, respectively. The 12-month PFS and OS were $23.8 \%$ and $37.7 \%$, respectively. The phase II TRINITY study evaluated the efficacy and safety of rovalpituzumab tesirine as a third- or further-line therapy for patients with ES-SCLC and delta-like 3 protein expression. For the entire cohort, the ORR was $12.4 \%$ and the median OS was 5.6 months. However, grade III-V adverse events were observed in $63 \%$ of patients (36).

Anlotinib is a novel multi-target inhibitor of tumor angiogenesis and growth that is approved as a third-line treatment for ES-SCLC by the Chinese Food and Drug Administration (CFDA) (37). In the ALTER1202 study, the median PFS of the anlotinib group exceeded that of the placebo group (4.3 vs. 0.7 months, $\mathrm{HR}=0.19, \mathrm{P}<0.0001$ ). The median OS in the anlotinib group reached 7.3 months, compared with 4.9 months in the placebo group (1).

The results of a retrospective study designed to investigate the efficacy of paclitaxel as the third-line chemotherapy for SCLC showed that the median PFS and OS were 2.5 and 5.9 months, respectively, and the incidence of grade III or IV neutropenia and thrombocytopenia was $20 \%$ and $10 \%$, respectively.

In summary, the survival benefits observed in our data are comparable to those reported by previous studies of patients with ES-SCLC in the third- or further-line setting. Our findings suggest that apatinib with oral etoposide is effective with good tolerability and could potentially serve as a third- or further-line therapy for patients with ESSCLC. However, we acknowledge that our study is a singlearm clinical trial and lacks a control group for comparison; therefore, selection bias cannot be ruled out. Nevertheless, our study demonstrated the efficacy and safety of lowdose apatinib combined with oral etoposide as a third- or further-line treatment for patients with ES-SCLC. Our results offer a manageable and feasible treatment strategy for ES-SCLC patients who have been heavily pretreated and provides further evidence for the clinical usage of the combination of low-dose apatinib and etoposide in ESSCLC. Furthermore, apatinib and etoposide capsules can be taken orally and do not require intravenous access or hospitalization; therefore, they may offer a useful, convenient, and compassionate treatment option for patients and their families in the palliative setting.

\section{Conclusions}

Overall, the present analysis has demonstrated that the administration of apatinib combined with orally administrated etoposide as a third- or further-line treatment exhibited promising survival benefits in patients with ESSCLC. Considering its encouraging efficacy and reasonable 
toxicity, apatinib with oral etoposide may provide a more economical and convenient treatment alternative for patients with ES-SCLC in a similar setting. However, our findings are limited by the small sample size and lack of control subjects; confirmation in a well-designed phase III clinical trial is necessary.

\section{Acknowledgments}

We would like to thank the patients and their families who participated in this study, and we also thank Jiangsu Hengrui Pharmaceuticals Co. for offering a discount on apatinib. The project was cosponsored by the Henan Province and Ministry of Health, Medical Science and Technology Program (No. 201601026) and in part by the National Natural Science Foundation of China (No. 81272600), Natural Science Foundation of Henan Province (No. 162300410300), ZHONGYUAN QIANREN JIHUA, and the 51282 Projects Leading Talent of Henan Provincial Health Science and Technology Innovation Talents (No. [2016]32). The program also received support from Science and Technology Innovation Talents in Universities of Henan Province (No. 18HASTIT044).

\section{Footnote}

Reporting Checklist: The authors have completed the TREND reporting checklist. Available at http://dx.doi. org/10.21037/tlcr-20-1235

Data Sharing Statement: Available at http://dx.doi. org/10.21037/tlcr-20-1235

Conflicts of Interest: All authors have completed the ICMJE uniform disclosure form (available at http://dx.doi. org/10.21037/tlcr-20-1235). The authors have no conflicts of interest to declare.

Ethical Statement: The authors are accountable for all aspects of the work in ensuring that questions related to the accuracy or integrity of any part of the work are appropriately investigated and resolved. The independent ethics committee of Henan Cancer Hospital of Zhengzhou University granted approval for this study, which was carried out in adherence to the Declaration of Helsinki (as revise in 2013) and Good Clinical Practice Guidelines. All patients provided written informed consent.
Open Access Statement: This is an Open Access article distributed in accordance with the Creative Commons Attribution-NonCommercial-NoDerivs 4.0 International License (CC BY-NC-ND 4.0), which permits the noncommercial replication and distribution of the article with the strict proviso that no changes or edits are made and the original work is properly cited (including links to both the formal publication through the relevant DOI and the license). See: https://creativecommons.org/licenses/by-nc-nd/4.0/.

\section{References}

1. Yang S, Zhang Z, Wang Q. Emerging therapies for small cell lung cancer. J Hematol Oncol 2019;12:47.

2. Liu $\mathrm{Y}, \mathrm{Hu} \mathrm{X}$, Jiang J, et al. A prospective study of apatinib in patients with extensive-stage small cell lung cancer after failure of two or more lines of chemotherapy. Oncologist 2020;25:e833-e842.

3. van Meerbeeck JP, Fennell DA, De Ruysscher DK. Smallcell lung cancer. Lancet 2011;378:1741-55.

4. Gazdar AF, Bunn PA, Minna JD. Small-cell lung cancer: what we know, what we need to know and the path forward. Nat Rev Cancer 2017;17:725-37.

5. Schneider BJ. Management of recurrent small cell lung cancer. J Natl Compr Canc Netw 2008;6:323-31.

6. Eckardt JR, von Pawel J, Pujol JL, et al. Phase III study of oral compared with intravenous topotecan as secondline therapy in small-cell lung cancer. J Clin Oncol 2007;25:2086-92.

7. Fontanini G, Faviana P, Lucchi M, et al. A high vascular count and overexpression of vascular endothelial growth factor are associated with unfavourable prognosis in operated small cell lung carcinoma. Br J Cancer 2002;86:558-63.

8. Pujade-Lauraine E, Hilpert F, Weber B, et al. Bevacizumab combined with chemotherapy for platinum-resistant recurrent ovarian cancer: The AURELIA open-label randomized phase III trial. J Clin Oncol 2014;32:1302-8.

9. Mo M, Yang J, Zhu X, et al. Bevacizumab Maintenance in Metastatic Colorectal Cancer. J Clin Oncol 2018;36:2451-2.

10. Zhu YJ, Zhang HB, Liu YH, et al. Meta-analysis of the role of bevacizumab in extensive stage small cell lung cancer. Oncol Lett 2017;14:655-64.

11. Spigel DR, Townley PM, Waterhouse DM, et al. Randomized phase II study of bevacizumab in combination with chemotherapy in previously untreated extensive-stage 
small-cell lung cancer: results from the SALUTE trial. J Clin Oncol 2011;29:2215-22.

12. Pujol JL, Lavole A, Quoix E, et al. Randomized phase II-III study of bevacizumab in combination with chemotherapy in previously untreated extensive small-cell lung cancer: results from the IFCT-0802 trialt. Ann Oncol 2015;26:908-14.

13. Tiseo M, Boni L, Ambrosio F, et al. Italian, Multicenter, Phase III, Randomized Study of Cisplatin Plus Etoposide With or Without Bevacizumab as First-Line Treatment in Extensive-Disease Small-Cell Lung Cancer: The GOIRC-AIFA FARM6PMFJM Trial. J Clin Oncol 2017;35:1281-7.

14. Xu Y, Huang $\mathrm{Z}, \mathrm{Lu} \mathrm{H}$, et al. Apatinib in patients with extensive-stage small-cell lung cancer after secondline or third-line chemotherapy: a phase II, singlearm, multicentre, prospective study. Br J Cancer 2019;121:640-6.

15. Scott LJ. Apatinib: A Review in Advanced Gastric Cancer and Other Advanced Cancers. Drugs 2018;78:747-58.

16. Gandara DR, Paul SM, Kowanetz M, et al. Bloodbased tumor mutational burden as a predictor of clinical benefit in non-small-cell lung cancer patients treated with atezolizumab. Nat Med 2018;24:1441-8.

17. Pietanza MC, Byers LA, Minna JD, et al. Small cell lung cancer: will recent progress lead to improved outcomes? Clin Cancer Res 2015;21:2244-55.

18. Einhorn LH. Daily oral etoposide in the treatment of cancer. Semin Oncol 1991;18:43-7.

19. Lan CY, Wang Y, Xiong Y, et al. Apatinib combined with oral etoposide in patients with platinum-resistant or platinum-refractory ovarian cancer (AEROC): a phase 2, single-arm, prospective study. Lancet Oncol 2018;19:1239-46.

20. Hu X, Zhang J, Xu B, et al. Multicenter phase II study of apatinib, a novel VEGFR inhibitor in heavily pretreated patients with metastatic triple-negative breast cancer. Int J Cancer 2014;135:1961-9.

21. Du Y, Cao Q, Jiang C, et al. Effectiveness and safety of low-dose apatinib in advanced gastric cancer: A real-world study. Cancer Med 2020;9:5008-14.

22. Yang $\mathrm{G}, \mathrm{Xu} \mathrm{H}$, Yang L, et al. Apatinib in combination with pemetrexed-platinum chemotherapy for chemo-naive nonsquamous non-small cell lung cancer: a phase II clinical study. Lung Cancer 2020;147:229-36.

23. Arrieta O, Lara-Mejía L, Zatarain-Barrón ZL. Carboplatin plus etoposide or topotecan for small-cell lung cancer.
Lancet Oncol 2020;21:1132-4.

24. Eberhardt WE, Mitchell A, Crowley J, et al. The IASLC Lung Cancer Staging Project: Proposals for the Revision of the M Descriptors in the Forthcoming Eighth Edition of the TNM Classification of Lung Cancer. J Thorac Oncol 2015;10:1515-22.

25. Bernhardt EB, Jalal SI. Small Cell Lung Cancer. Cancer Treat Res 2016;170:301-22.

26. Le Pechoux C, Levy A. Radiosurgery in patients with small cell lung cancer with brain metastases: a call for prospective evidence. JAMA Oncol 2020;6:1037-8.

27. Iams WT, Porter J, Horn L. Immunotherapeutic approaches for small-cell lung cancer. Nat Rev Clin Oncol 2020;17:300-12.

28. Chen H, Horita N, Ito K, et al. Hazard ratio of progression-free survival is an excellent predictor of overall survival in phase III randomized controlled trials evaluating the first-line chemotherapy for extensivedisease small-cell lung cancer. Transl Lung Cancer Res 2020;9:1333-42.

29. Cheng S, Evans WK, Stys-Norman D, et al. Chemotherapy for relapsed small cell lung cancer: a systematic review and practice guideline. J Thorac Oncol 2007;2:348-54.

30. Liu C, Jia Q, Wei H, et al. Apatinib in patients with advanced chordoma: a single-arm, single-centre, phase 2 study. Lancet Oncol 2020;21:1244-52.

31. Cui Y, Cao Y, Cao M, et al. Efficacy and safety of apatinib monotherapy in metastatic renal cell carcinoma (mRCC) patients: A single-arm observational study. Urol Oncol 2020;38:936.e1-936.e6.

32. Yu Z, Cai X, Xu Z, et al. Apatinib plus chemotherapy as a second-line treatment in unresectable non-small cell lung carcinoma: a randomized, controlled, multicenter clinical trial. Oncologist 2020;25:e1640-9.

33. Rezonja R, Knez L, Cufer T, Mrhar A. Oral treatment with etoposide in small cell lung cancer - dilemmas and solutions. Radiol Oncol 2013;47:1-13.

34. Chen H, Wu X, Wang W, Wang Q. When cancer encounters COVID-19 in China: what have we suffered, experienced and learned. Jpn J Clin Oncol 2020;50:712-7.

35. Ready N, Farago AF, de Braud F, et al. Third-Line Nivolumab Monotherapy in Recurrent SCLC: CheckMate 032. J Thorac Oncol 2019;14:237-44.

36. Morgensztern D, Besse B, Greillier L, et al. Efficacy and Safety of Rovalpituzumab Tesirine in ThirdLine and Beyond Patients with DLL3-Expressing, 
Relapsed/Refractory Small-Cell Lung Cancer: Results From the Phase II TRINITY Study. Clin Cancer Res 2019;25:6958-66.

Cite this article as: $\mathrm{He} Z$, Zhou $\mathrm{H}$, Wang J, Li D, Zhang X, Wang P, Ma T, Zhang Y, Tian C, Chen Y, Zou M, Han Y, Xu C, Ma S, Wang L, Wu X, Chen G, Wang Q. Apatinib with etoposide capsules as a third- or further-line therapy for extensive-stage small cell lung cancer: an openlabel, multicenter, single-arm phase II trial. Transl Lung Cancer Res 2021;10(2):889-899. doi: 10.21037/tlcr-20-1235
37. Shen G, Zheng F, Ren D, et al. Anlotinib: a novel multitargeting tyrosine kinase inhibitor in clinical development. J Hematol Oncol 2018;11:120. 